\title{
Associations of fractional pulse pressure to aortic stiffness and their impact on diastolic function and coronary flow reserve in asymptomatic diabetic patients with normal coronary angiography
}

\author{
Ragab A. Mahfouz, Waleed Elawady, Mohamed Abdu, Abdelhakem Salem \\ Cardiology Department, Faculty of Medicine, Zagazig University, Egypt
}

\begin{abstract}
Background: We aimed to assess the relation of fractional pulse pressure (PPf) to aortic stiffness index and their impact on coronary flow reserve (CFR) and left ventricular diastolic function in asymptomatic diabetic patients.

Methods: One hundred and thirty five consecutive asymptomatic diabetic patients (aged $48.8 \pm$ \pm 7.84 years), were included. CFR was calculated noninvasively using transthoracic echo-Doppler assessment with hyperemia induced by infusion of dipyridamole at a rate of $0.56 \mathrm{mg} / \mathrm{kg}$ over 4 min. PPf was calculated as pulse pressure divided by mean arterial pressure (SBP-DBP/ $(M A P)$, while diastolic function was evaluated by means of transmitral flow and tissue Doppler imaging. Aortic stiffness indices (ASI) were measured as previously described.
\end{abstract}

Results: Diabetic patients with low CFR $(n=52)$ compared with those with normal CFR $(n=83)$ exhibited significantly increased PPf $(75.2 \pm 11.4$ vs. $64.5 \pm 6.7, p<0.001)$. PPf was significantly correlated with ASI $(r=0.520, p<0.001), E / E m$ ratio $(r=0.425$, $p<0.001)$ and left atrial volume index $(r=0.462, p<0.001)$. CFR was negatively correlated with both PPf $(r=-0.68, p<0.0001)$. After applying multivariate linear regression analysis, after correction for cardiovascular risk factors, importantly, PPf and ASI remained significant predictors of CFR $(p<0.0001$ and $p<0.001$, respectively).

Conclusions: PPf was significantly correlated to ASI in asymptomatic diabetic patients. Likewise, increased PPf was associated with impaired CFR and subclinical diastolic dysfunction in diabetic patients. PPf could be utilized as a simple non-invasive predictor of occult atherosclerosis and diastolic dysfunction in diabetic patients. (Cardiol J 2013; 20, 6: 605-611)

Key words: pulse pressure, diabetes mellitus, diastolic function, coronary flow

\section{Introduction}

Coronary artery disease (CAD) constitutes a major cause of morbidity and mortality in patients with diabetes mellitus. In addition, because of diabetic neuropathy, myocardial ischemia and infarction may be prevalent in the absence of typical anginal symptoms. Non-invasive assessment of $\mathrm{CAD}$ in asymptomatic diabetic patients at higher risk has therefore been previously suggested by the guidelines of the American Diabetes Association and the American Heart Association [1]. If

Address for correspondence: Ragab A. Mahfouz, MD, PhD, Cardiology Department, Faculty of Medicine, Zagazig University, Algamah Hospital, 12345 Zagazig, Egypt, tel: 00201006427671, e-mail: ragabmahfouz61@yahoo.com 
myocardial perfusion abnormalities are identified, coronary angiography is considered to confirm the presence of clinically relevant obstructive CAD possibly requiring intervention. However, previous studies have shown a discrepancy between the presence of myocardial perfusion defects and obstructive $\mathrm{CAD}$ in diabetic patients. In approximately $20-40 \%$ of diabetic patients, myocardial perfusion defects could not be clarified by obstruction of the coronary arteries [2, 3]. Abnormal coronary flow reserve (CFR) is often present in patients with cardiovascular risk factors despite the presence of angiographically normal coronary arteries [4].

We hypothesized that fractional pulse pressure (PPf) is thought to reflect arterial stiffness than pulse pressure, because dividing by mean arterial pressure theoretically cancels out the influence of cardiac output and peripheral vascular resistance.

The current study was designed to prospectively evaluate the relationship between PPf and aortic stiffness and their impact on asymptomatic diabetic patients with abnormal CFR, without obstructive CAD.

\section{Methods}

Prospectively, 135 asymptomatic diabetic patients were screened for cardiovascular disease. A structured interview, physical examination, and laboratory analysis were performed. Cardiovascular risk factors were assessed according to the following criteria. A family history of CAD was considered positive if CAD was present in any first-degree family member. Diabetes was defined as a fasting blood glucose level of $>126 \mathrm{mg} / \mathrm{dL}$ or treatment with dietary modification or oral hypoglycemic agents at the time of the study. Hypercholesterolemia was defined as fasting serum total cholesterol $>200 \mathrm{mg} / \mathrm{dL}$ or if the subject was being treated with lipid-lowering medication or dietary modification. Hypertension was defined as a seated systolic blood pressure $(\mathrm{SBP})>140 \mathrm{~mm} \mathrm{Hg}$ or diastolic blood pressure (DBP) $>90 \mathrm{~mm} \mathrm{Hg}$ on at least 3 occasions, or if such a diagnosis had been made in the past and the patient was treated with medications or lifestyle modification. Body mass index was calculated as weight $[\mathrm{kg}]$ divided by height $\left[\mathrm{m}^{2}\right]$. All patients with angiographically documented CAD were excluded from the study.

CFR and coronary angiography were performed as part of the clinical work-up of all patients. Concurrent measurements of PPf aortic stiffness indices (ASI) were performed.
The local ethical committee approved this study. Informed consent was obtained from all patients.

\section{Blood pressure measurement}

Blood pressure measurement was performed at 3 different visits in our outpatient clinic by using a mercury sphygmomanometer with the patient in the sitting position resting for at least $5 \mathrm{~min}$. Pulse pressure was calculated as the difference between SBP and DBP. Mean arterial pressure was calculated as DBP plus one third of pulse pressure. PPf was calculated according to the following equation: fractional pulse pressure $=$ systolic blood pressure - diastolic blood pressure/mean arterial pressure $(\mathrm{PPf}=\mathrm{SBP}-\mathrm{DBP} / \mathrm{MAP})$

\section{Evaluation of coronary flow reserve}

Left anterior descending (LAD) coronary artery was visualized using a modified, foreshortened 2 -chamber view, and an optimal alignment to the interventricular sulcus was obtained. The color gain was adjusted to provide optimal images and coronary flow in the distal LAD was examined by color Doppler flow mapping over the epicardial part of the anterior wall. Pulsed wave Doppler recordings of the mid-to-distal LAD coronary artery were obtained from each subject. Spectral Doppler of the LAD coronary artery displayed a characteristic biphasic flow pattern, with a larger diastolic and a smaller systolic component. Hyperemia was induced by infusion of dipyridamole at a rate of $0.56 \mathrm{mg} / \mathrm{kg}$ over $4 \mathrm{~min}$. Coronary peak diastolic velocities were measured at baseline and at hyperemia. The highest 3 Doppler recordings were averaged for each measurement. CFR was calculated as the ratio of hyperemic to baseline peak diastolic velocities [5]. CFR is a method of assessing the functional severity of coronary stenosis [6]. The exact cut-off value that divides "normal" from "abnormal" CFR - i.e. microcirculation dysfunction - is rather vague. The recent CFR consensus [6] considers a value of $<2.0$ a proof of ischemia and of hemodynamically significant stenosis, and thus an indication for intervention. Values 2.0-2.5 are the so-called grey zone, and only values $>2.5$ are considered completely normal. On this basis, we used a CFR cut-off value of 2.5 to divide the diabetic patients into those with normal $(>2.5)$ and low $(\leq 2.5)$ CFR.

\section{Noninvasive evaluation of aortic stiffness}

The transverse displacement of the aortic wall was measured with commercially available 
equipment (GE-Vivid 3; General Electric, Milwaukee, WI) with 2.5 and $3.5 \mathrm{MHz}$ transducers. After routine conventional echocardiographic examination, patients were placed in a left mild recumbent position and the ascending aorta was recorded in the 2-dimensional guided $\mathrm{M}$-mode tracings. The aortic diameter was recorded by M-mode echocardiogram at a level of $3 \mathrm{~cm}$ above the aortic valve. Internal aortic diameters were measured by means of a caliper in systole and diastole as the distance between the trailing edge of the anterior aortic wall and the leading edge of the posterior aortic wall. Aortic systolic (AoS) diameter was measured at the time of full opening of the aortic valve and diastolic (AoD) diameter was measured at the peak of QRS complex of the ECG. Five consecutive beats were measured routinely and averaged. Measurements were performed by the same investigator; the intraobserver variability in aortic diameter measurements was $2.2 \%$. The obtained values were used to calculate aortic stiffness indices:

Aortic strain $($ AOST) $=$ systolic diameter $(\mathrm{SD})$ - diastolic diameter $(\mathrm{DD}) \times 100 /$ diastolic diameter (DD); and elastic modulus E(p) $=\mathrm{PP} /$ strain. Aortic stiffness (b) Index = In (SBP/DBP)/(AoS - AoD)/ /AoD (pure number), and aortic distensibility $\left(\mathrm{cm}^{2} /\right.$ $/$ dyn $)=2 \times$ AOST/pulse pressure [7-9].

\section{Statistical analysis}

All analyses were performed using commercially-available statistical software (Statistical Package for the Social Sciences [SPSS], version 13.0; SPSS Inc., Chicago, IL, USA). A p less than 0.05 was considered to indicate statistical significance.

\section{Results}

Table 1 represents the demographic characteristics of the study population. With respect to CFR measurements, and based on a CFR cutoff value of 2.5 diabetic patients were classified into those with low $(\mathrm{n}=52)$ CFR and normal CFR $(\mathrm{n}=83)$ (Table 2). Diabetic patients with low CFR compared with
Table 1. Characteristics of study population (135 patients).

\begin{tabular}{lc}
\hline Characteristic & Value \\
\hline Age [years] & $52 \pm 13$ \\
Male sex & $58 \%$ \\
Diabetes mellitus duration [years] & $13 \pm 9$ \\
Body mass index $\left[\mathrm{kg} / \mathrm{m}^{2}\right]$ & $26 \pm 5$ \\
Hemoglobin A1c $[\mathrm{mmol} / \mathrm{L}]$ & $7.1 \pm 1.4$ \\
Smoking & $27 \%$ \\
Family history of coronary & $49 \%$ \\
artery disease & \\
Hypercholesterolemia & $63 \%$ \\
Hypertension & $41 \%$ \\
Diabetes treatment: & \\
Diet only & $0 \%$ \\
Oral agent & $55 \%$ \\
Oral agent and insulin & $23 \%$ \\
Insulin & $22 \%$ \\
\hline
\end{tabular}

those with normal CFR did not differ with respect to age, sex, body mass index, smoking incidence, SBP, metabolic profile and serum creatinine levels ( $p>0.05$ for all cases). The significant differences were observed in $\operatorname{PPf}(\mathrm{p}<0.0001), \operatorname{DBP}(\mathrm{p}<0.05)$ and pulse pressure $(\mathrm{p}<0.05)$ (Table 3$)$.

As far as echocardiographic parameters are concerned, diabetic patients with low CFR compared with those with normal CFR had increased left atrial volume index $(\mathrm{p}<0.03)$. Moreover, diabetic patients with low CFR compared with those with normal CFR were characterized by significantly increased $\mathrm{E} / \mathrm{Em}$ ratio $(\mathrm{p}<0.001)$ (Table 4).

The results showed that in diabetic patients, PPf\% was significantly correlated to the increased ASI $(\mathrm{r}=0.52, \mathrm{p}<0.001)$ (Fig. 1), with $\mathrm{E} / \mathrm{Em}(\mathrm{r}=0.425, \mathrm{p}<0.001)$ (Fig. 2), and left atrial volume index $(r=0.462, \mathrm{p}<0.003)$. On the other hand, PPf was negatively correlated with CFR ( $r=-0.68, p<0.0001)$ (Fig. 3, Table 5). After applying multivariate linear regression analysis,

Table 2. Coronary blood flow characteristics findings for diabetic patients with low and normal coronary flow reserve (CFR).

\begin{tabular}{lccc}
\hline & $\begin{array}{c}\text { Diabetics with low CFR } \\
(\mathbf{n}=\mathbf{5 2})\end{array}$ & $\begin{array}{c}\text { Diabetics normal CFR } \\
\text { (n= 83) }\end{array}$ & P \\
\hline Baseline average peak velocity & $25.2 \pm 1.6$ & $29.8 \pm 2.9$ & $>0.05$ \\
Baseline diastolic to systolic velocity ratio & $1.72 \pm 0.52$ & $1.85 \pm 0.41$ & $>0.05$ \\
Peak average peak velocity & $60.5 \pm 15.2$ & $75.1 \pm 11.4$ & $<0.05$ \\
CFR & $1.8 \pm 0.21$ & $3.23 \pm 0.29$ & $<0.001$ \\
\hline
\end{tabular}


Table 3. Clinical characteristics and laboratory findings for diabetics with low and normal coronary flow reserve (CFR).

\begin{tabular}{lccc}
\hline & Diabetics with low CFR $\mathbf{n}=\mathbf{5 2})$ & Diabetics normal CFR $\mathbf{n}=\mathbf{8 3})$ & $\mathbf{P}$ \\
\hline Age [year] & $53.8 \pm 9.6$ & $53.1 \pm 11.3$ & $>0.05$ \\
Gender (males) & $76.5 \%$ & $72.8 \%$ & $>0.05$ \\
Body mass index $\left[\mathrm{kg} / \mathrm{m}^{2}\right]$ & $26.6 \pm 3.9$ & $25.3 \pm 4.2$ & $>0.05$ \\
Smokers & $38.2 \%$ & $37.5 \%$ & $>0.05$ \\
Systolic BP & $142.3 \pm 13$ & $139.2 \pm 14$ & $>0.05$ \\
Diastolic BP & $72.3 \pm 12$ & $80.2 \pm 15$ & $<0.05$ \\
Pulse pressure & $71.0 \pm 5.7$ & $60.5 \pm 8.4$ & $<0.05$ \\
Mean atrial pressure & $95.2 \pm 8$ & $98.1 \pm 7$ & $>0.05$ \\
PPf\% & $73.2 \pm 7$ & $60.4 \pm 5$ & $<0.001$ \\
Total cholesterol [mg/dL] & $189.3 \pm 48$ & $185.2 \pm 42$ & 0.60 \\
HDL cholesterol [mg/dL] & $49.4 \pm 15$ & $48.8 \pm 17$ & 0.44 \\
LDL cholesterol [mg/dL] & $149.8 \pm 32$ & $142.1 \pm 31$ & 0.42 \\
Triglycerides [mg/dL] & $145.5 \pm 42$ & $132.5 \pm 39$ & 0.69 \\
Glucose [mg/dL] & $114.5 \pm 16$ & $98.5 \pm 14$ & 0.81 \\
Serum creatinine [mg/dL] & $0.89 \pm 0.13$ & $0.91 \pm 0.13$ & 0.17 \\
\hline
\end{tabular}

BP — blood pressure; PPf — fractional pulse pressure; HDL and LDL — high and low-density lipoprotein

Table 4. Echocardiographic parameters for diabetics with low and normal coronary flow reserve (CFR).

\begin{tabular}{|c|c|c|c|}
\hline & $\begin{array}{l}\text { Diabetics with low CFR } \\
\qquad(\mathrm{n}=52)\end{array}$ & $\begin{array}{l}\text { Diabetics normal CFR } \\
\qquad(\mathrm{n}=\mathbf{8 3})\end{array}$ & $\mathbf{P}$ \\
\hline Left ventricular mass index $\left[\mathrm{g} / \mathrm{m}^{2}\right]$ & $71.2 \pm 15$ & $70.3 \pm 11$ & $>0.01$ \\
\hline Relative wall thickness & $0.40 \pm 0.05$ & $0.39 \pm 0.04$ & $>0.05$ \\
\hline Left atrial volume index $\left[\mathrm{mL} / \mathrm{m}^{2}\right]$ & $26.5 \pm 3.7$ & $19.0 \pm 3.1$ & $<0.03$ \\
\hline$E[\mathrm{~ms}]$ & $0.69 \pm 0.13$ & $0.77 \pm 0.16$ & $>0.05$ \\
\hline $\mathrm{A}[\mathrm{ms}]$ & $0.71 \pm 0.12$ & $0.62 \pm 0.11$ & $>0.05$ \\
\hline $\mathrm{E} / \mathrm{A}$ & $0.92 \pm 0.15$ & $1.15 \pm 0.15$ & $<0.05$ \\
\hline Isovolumic relaxation time [ms] & $123 \pm 28$ & $109 \pm 22$ & $>0.05$ \\
\hline Deceleration time [ms] & $264 \pm 412$ & $269 \pm 33$ & $>0.05$ \\
\hline $\mathrm{Em}[\mathrm{cm} / \mathrm{s}]$ & $6.3 \pm 1.2$ & $9.8 \pm 1.7$ & $<0.02$ \\
\hline $\mathrm{Am}[\mathrm{cm} / \mathrm{s}]$ & $11.5 \pm 1.5$ & $8.1 \pm 1.2$ & $>0.05$ \\
\hline $\mathrm{Em} / \mathrm{Am}$ & $0.69 \pm 0.12$ & $0.91 \pm 0.23$ & $<0.05$ \\
\hline $\mathrm{E} / \mathrm{Em}$ & $11.2 \pm 1.3$ & $7.8 \pm 1.1$ & $<0.001$ \\
\hline
\end{tabular}

$\mathrm{E}$ and $\mathrm{A}$ - peak early and late transmitral flow velocities; Em and Am — peak early and late myocardial diastolic velocities

$\operatorname{PPf}(\mathrm{p}<0.0001), \operatorname{ASI}(\mathrm{p}<0.002), \mathrm{E} / \mathrm{Am}(\mathrm{p}<0.02)$, turned to be the most powerful independent predictors of impaired CFR (Table 6).

\section{Discussion}

The current prospective study revealed that $38.5 \%$ of asymptomatic patients with diabetes mellitus had abnormal CFR despite the absence of obstructive CAD. PPf was significantly correlated with ASI. PPf and ASI were significantly higher in patients with abnormal CFR than in those with normal CFR. Further, both PPf and ASI were significantly correlated with left ventricular dysfunction. Importantly, after correction for risk factors, PPf and ASI remained significant predictors of abnormal CFR.

It is not surprising that pulse pressure is associated with an increased risk of cardiovascular events (MI, CHF) and death [10, 11]. However, because different combinations of SBP and DBP give the same pulse pressure value (e.g., blood pressures of 130/60 and 180/110 mm Hg both give a pulse pressure of $70 \mathrm{~mm} \mathrm{Hg}$ ), indeed, $\mathrm{PPf}$, repre- 


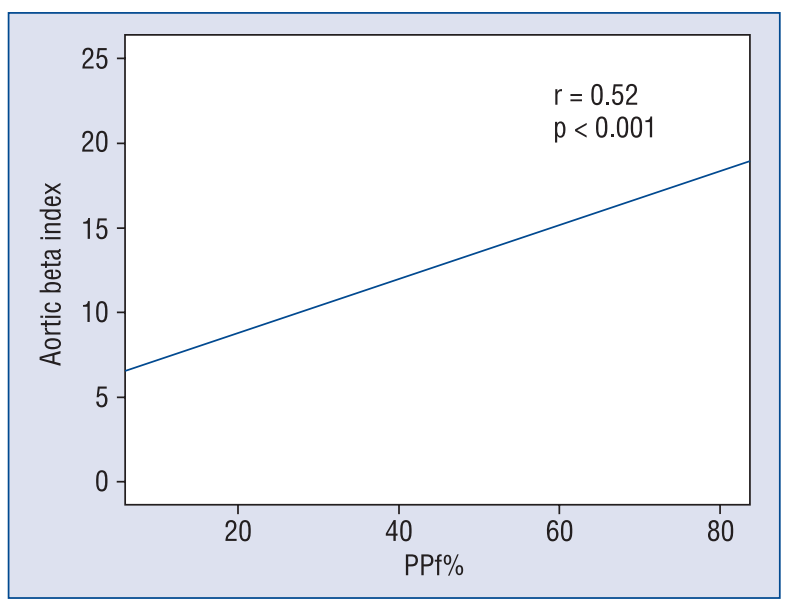

Figure 1. Linear regression analysis and Pearson correlation coefficient for the relationship between fractional pulse pressure (PPf)\% and aortic beta index.

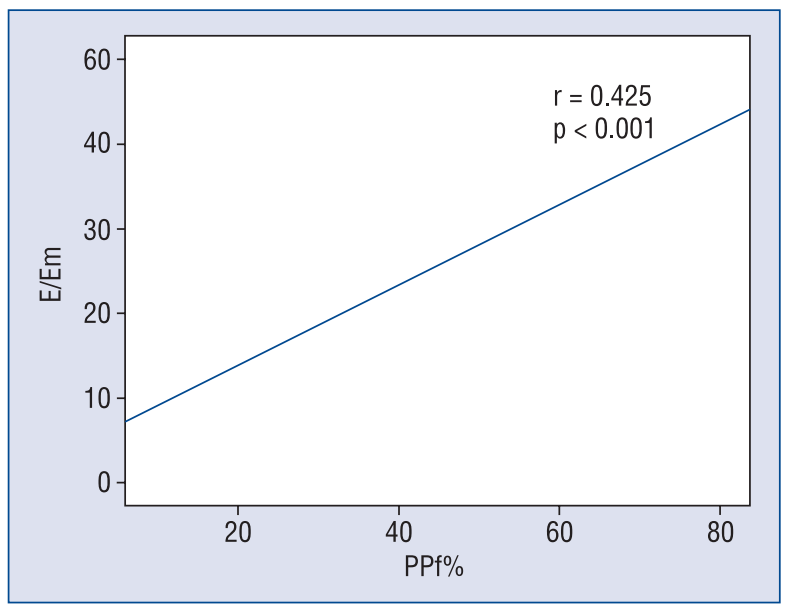

Figure 2. Linear regression analysis and Pearson correlation coefficient for the relationship between fractional pulse pressure (PPf) \% and E/Em.

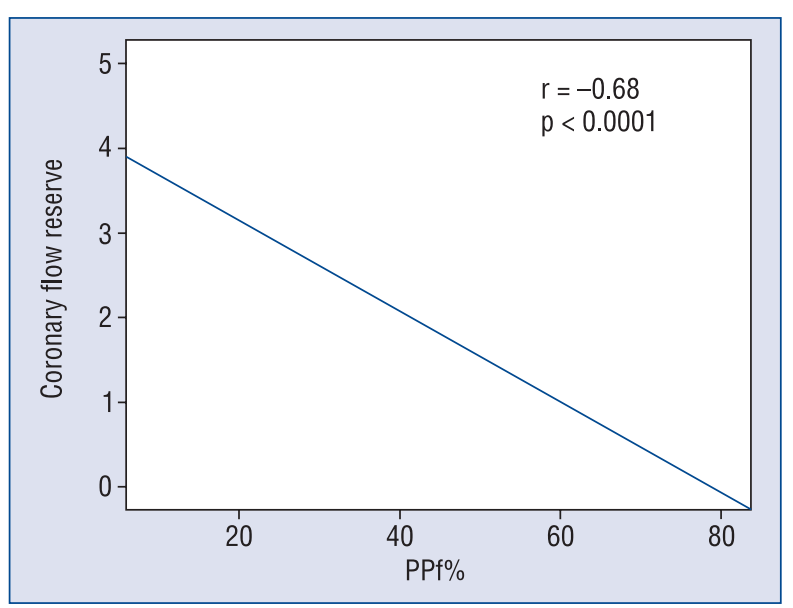

Figure 3. Linear regression analysis and Pearson correlation coefficient for the relationship between fractional pulse pressure (PPf) \% and coronary flow reserve.
Table 5. Pearson's linear correlation between fractional pulse pressure and study variables.

\begin{tabular}{lcc}
\hline Variable & $\mathbf{r}$ & $\mathbf{p}$ \\
\hline Age & 0.243 & $>0.05$ \\
Male sex & 0.147 & $>0.05$ \\
Body mass index $\left[\mathrm{kg} / \mathrm{m}^{2}\right]$ & 0.253 & $>0.05$ \\
Hypercholesterolemia & 0.211 & $>0.05$ \\
Left ventricular ejection & 0.175 & $>0.05$ \\
fraction [\%] & & \\
Left atrial volume & 0.462 & $<0.001$ \\
index [mL/m $\left.{ }^{2}\right]$ & & \\
E/Em & 0.425 & $<0.02$ \\
Aortic stiffness beta index & 0.520 & $<0.001$ \\
Coronary flow reserve & -0.690 & $<0.0001$ \\
\hline
\end{tabular}

Table 6. Independent predictors of coronary flow reserve in multiple linear regression analysis.

\begin{tabular}{lcc}
\hline Variable & HR $(95 \% \mathrm{Cl})$ & $\mathbf{P}$ \\
\hline Age & $1.02(0.85-1.27)$ & $>0.05$ \\
SBP & $0.992(0.82-1.22)$ & $>0.05$ \\
Hypercholesterolemia & $0.893(0.65-1.15)$ & $>0.05$ \\
Triglyceride & $1.015(0.79-1.17)$ & $>0.05$ \\
Smoking & $0.895(0.81-1.02)$ & $>0.05$ \\
E/A ratio & $0.96(0.64-1.52)$ & $>0.05$ \\
E/Am ratio & $1.78(1.08-2.45)$ & 0.02 \\
PPf (\%) & $2.25(1.22-3.05)$ & 0.0001 \\
Aortic stiffness index & $1.84(1.22-3.05)$ & 0.001 \\
\hline
\end{tabular}

$\mathrm{Cl}$ - confidence interval; $\mathrm{E}$ and $\mathrm{A}$ - peak early and late transmitral flow velocities; $\mathrm{Em}$ and $\mathrm{Am}$ - peak early and late myocardial diastolic velocities; HR — hazard ratio; SBP — systolic blood pressure; PPf — fractional pulse pressure

sented as a parameter of the pulsatile component of blood pressure, was superior to pulse pressure in terms of the risk stratification of atherosclerosis. We evaluated the relation between PPf and CFR in diabetic patients with normal coronary arteries.

Diabetes is one of the most important risk factors for the presence of aortic atherosclerotic plaque, and a positive association has been shown between the presence of atherosclerotic plaque and arterial stiffness [12]. It is important to note that some of the mechanisms by which arteries stiffness after exposure to elevations in blood glucose occur rapidly, whereas others require more time to develop. For example, peripheral arterial endothelial function is known to deteriorate $1 \mathrm{~h}$ after high glucose oral intake while increases the local activity of renin-angiotensin-aldosterone system and expression of angiotensin type 1 receptor in 
vascular tissue promoting development of wall hypertrophy and fibrosis are chronic mechanisms [13].

A study of asymptomatic patients with risk factors for ischemic heart disease showed that patients with higher augmentation index and pulse wave velocity had a lower CFR. In a normal elastic aorta the pulse wave reflects from the periphery and returns to the heart in diastole, which improves the diastolic filling of the coronary arteries. With aorta stiffening the pulse wave returns during systole resulting in increased afterload and myocardial oxygen demand which could mean that a reduced CFR in heart failure patients without significant coronary artery stenosis might not only be due to impaired coronary microcirculation, but that a reduced diastolic filling can be a participating factor $[14,15]$. Ascending aorta pulse and PPf are significantly associated with the presence of cardiac syndrome $\mathrm{X}$ and these associations are independent of age and other cardiovascular risk factors [16]. The increased aortic stiffness is regarded as a contributor to the development of increased systolic hypertension with reduced DBP and increased pulse pressure [17]. These changes contribute to the increased myocardial load and to the reduced myocardial oxygen balance [18].

The current study shows that there is a significant correlation between PPf and ASI with left ventricular diastolic function. An increase in PPf and aortic stiffness was directly associated with a significant increase in $\mathrm{E} / \mathrm{Em}$ ratio an indirect parameter of left ventricular filling pressure. Of note, these parameters have been shown to be associated with symptomatic status and poor prognosis [19].

Elevated left ventricular diastolic pressure associated with reduced arterial DBP limits the CFR and leads to subendocardial ischemia even in the absence of significant CAD [20]. Hence, the longitudinal function, governed by the subendocardial fibres, is the first to be altered, while left ventricular ejection fraction, depending more on mid-wall myocardial fibres, is maintained within the normal range until the compensatory mechanisms are exhausted [21, 22].

\section{Clinical implications}

In the current study, abnormal CFR not attributable to obstructive $\mathrm{CAD}$ could be related to impaired endothelial function. Increased aortic stiffness is usually associated with impaired endothelial function. Accordingly, increased PPf could be considered a simple noninvasive parameter of subclinical atherosclerosis in patients with $\mathrm{CAD}$ risk factors like diabetes mellitus; consequently, intensification of antiatherogenic therapy seems to be indicated in asymptomatic diabetic patients with abnormal CFR even in absence of obstructive CAD. Furthermore, considering that in diabetes mellitus abnormal CFR is often observed in the absence of obstructive $\mathrm{CAD}$, the question emerges of whether this test should be accompanied by a noninvasive test. Indeed, referral of all asymptomatic diabetic patients with abnormal CFR for invasive coronary angiography should be avoided considering that many patients will not have obstructive epicardial stenosis amenable to revascularization. The combined noninvasive assessment with PPf and ASI may allow a more comprehensive assessment of $\mathrm{CAD}$ and facilitate further management.

\section{Conclusions}

In asymptomatic diabetic patients with no obstructive epicardial coronary artery, increased PPf was an independent predictor of abnormal CFR and is strongly associated with increased ASI. In view of the likely reversible nature of endothelial dysfunction and the cardiovascular prognostic value of abnormal CFR in asymptomatic diabetic patients, it is presumable that PPf could be utilized as a noninvasive parameter in risk stratification of asymptomatic diabetic patients who will benefit from intensified pharmacologic treatment even in the absence of obstructive CAD.

\section{Conflict of interest: none declared}

\section{References}

1. Consensus development conference on the diagnosis of coronary heart disease in people with diabetes: 10-11 February 1998, Miami, Florida. American Diabetes Association. Diabetes Care, 1998; 21: 1551-1559.

2. Scholte AJ, Schuijf JD, Kharagiitsingh AV et al. Different manifestations of coronary artery disease by stress SPECT myocardial perfusion imaging, coronary calcium scoring, and multislice CT coronary angiography in asymptomatic patients with type 2 diabetes mellitus. J Nucl Cardiol, 2008; 15: 503-509.

3. Kang X, Berman DS, Lewin $\mathrm{H}$ et al. Comparative ability of myocardial perfusion single-photon emission computed tomography to detect coronary artery disease in patients with and without diabetes mellitus. Am Heart J, 1999; 137: 949-957.

4. Camici PG, Crea F. Coronary microvascular dysfunction. N Engl J Med, 2007; 356: 830-840.

5. Dimitrow PP, Galderisi M, Rigo F. The non-invasive documentation of coronary microcirculation impairment: Role of transthoracic echocardiography. Cardiovasc Ultrasound, 2005; 3: 18.

6. Kern MJ, Lerman A, Bech JW et al. Physiological assessment of coronary artery disease in the cardiac catheterization laboratory: A scientific statement from the American Heart Association Committee on Diagnostic and Interventional Cardiac Catheteri- 
zation, Council on Clinical Cardiology. Circulation, 2006; 114: 1321-1341.

7. Pitsavos C, Toutouzas K, Dernellis J et al. Aortic stiffness in young patients with heterozygous familial hypercholesterolemia. Am Heart J, 1998; 135: 604-608.

8. Mahfouz RA, Dewedar A, Abdelmoneim A et al. Aortic and pulmonary artery stiffness and cardiac function in children at risk for obesity. Echocardiography, 2012; 29: 984-990.

9. Lacombe F, Dart A, Dewar E et al. Arterial elastic properties in man: A comparison of echo-Doppler indices of aortic stiffness. Eur Heart J, 1992; 13: 1040-1045.

10. Benetos A, Rudnichi A, Safar M, Guize L. Pulse pressure and cardiovascular mortality in normotensive and hypertensive subjects. Hypertension, 1998; 32: 560-564.

11. Franklin SS, Khan SA, Wong ND, Larson MG, Levy D. Is pulse pressure useful in predicting risk for coronary heart disease? The Framingham heart study. Circulation, 1999; 100: 354-360.

12. Qureshi G, Brown R, Salciccioli L et al. Relationship between aortic atherosclerosis and non-invasive measures of arterial stiffness. Atherosclerosis, 2007; 195: e190- e194.

13. Lee IK, Kim HS, Bae JH. Endothelial dysfunction: Its relationship with acute hyperglycaemia and hyperlipidemia. Int J Clin Pract Suppl, 2002; 129: 59- 64.

14. Saito M, Okayama H, Nishimura K et al. Possible link between large artery stiffness and coronary flow velocity reserve. Heart, 2008; 94: e20.

15. Snoer M, Monk-Hansen T, Olsen RH et al. Insulin resistance and exercise tolerance in heart failure patients: Linkage to coronary flow reserve and peripheral vascular function. Cardiovascular Diabetology, 2012; 11: 97 (doi: 10.1186/1475-2840-11-97).

16. Cay S, Guray U, Korkmaz S. Increased aortic pulse and fractional pulse pressures in patients with cardiac syndrome X. Blood Press, 2006; 15: 179-184.

17. Dart AM, Kingwell BA. Pulse pressure: a review of mechanisms and clinical relevance. J Am Coll Cardiol, 2001; 37: 975-984.

18. London GM, Guerin AP. Influence of arterial pulse and reflected waves on blood pressure and cardiac function. Am Heart J, 1999; 138: $220-224$.

19. Lancellotti P, Donal E, Magne J et al. Risk stratification in asymptomatic moderate to severe aortic stenosis: The importance of the valvular, arterial and ventricular interplay. Heart, 2010; 96: 1364-1371.

20. Hein S, Arnon E, Kostin S et al. Progression from compensated hypertrophy to failure in the pressure-overloaded human heart: Structural deterioration and compensatory mechanisms. Circulation, 2003; 107: 984-991.

21. Bruch C, Stypmann J, Grude M, Gradaus R, Breithardt G, Wichter T Tissue Doppler imaging in patients with moderate to severe aortic valve stenosis: Clinical usefulness and diagnostic accuracy. Am Heart J, 2004; 148: 696-702.

22. Galema TW, Yap SC, Geleijnse ML et al. Early detection of left ventricular dysfunction by Doppler tissue imaging and $\mathrm{N}$-terminal pro-B-type natriuretic peptide in patients with symptomatic severe aortic stenosis. J Am Soc Echocardiogr, 2007; 21: 257-261. 